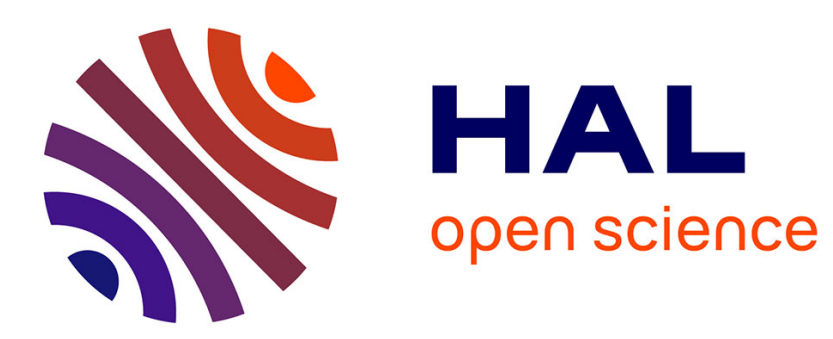

\title{
Optimization of Parametrised Kicking Motion for Humanoid Soccer Player
}

Nicolas Jouandeau, Vincent Hugel

\section{To cite this version:}

Nicolas Jouandeau, Vincent Hugel. Optimization of Parametrised Kicking Motion for Humanoid Soccer Player. IEEE International Conference on Autonomous Robot Systems and Competitions, May 2014, Espinho, Portugal. 10.1109/ICARSC.2014.6849793 . hal-02317147

\section{HAL Id: hal-02317147 https://hal.science/hal-02317147}

Submitted on 15 Oct 2019

HAL is a multi-disciplinary open access archive for the deposit and dissemination of scientific research documents, whether they are published or not. The documents may come from teaching and research institutions in France or abroad, or from public or private research centers.
L'archive ouverte pluridisciplinaire HAL, est destinée au dépôt et à la diffusion de documents scientifiques de niveau recherche, publiés ou non, émanant des établissements d'enseignement et de recherche français ou étrangers, des laboratoires publics ou privés. 


\title{
Optimization of Parametrised Kicking Motion for Humanoid Soccer Player
}

\author{
Nicolas Jouandeau ${ }^{a}$ and Vincent Hugel ${ }^{b}$
}

\begin{abstract}
This paper presents a detailed description of the optimization process used to increase the kicking skills of humanoid players. The kicking movement consists of making a step forward to put the support foot next to the ball to kick, then to execute the kicking motion by rocking the leg that must strike the ball. The rocking motion of the leg passes through three positions; the first position is the leg being raised backward, the second is the leg in position of strike, and the third is the leg forward to sweep the ball. The optimization process is an evolution of skills parameters guided by a evaluation function that aims to send the ball as far as possible. To optimize the kick a rocking motion of the torso is also introduced. The parameters used for each one of the three kicking positions are the angle of the torso, the angle of the foot sole, the longitudinal and the vertical coordinates of the foot toe. Thanks to this technique it is possible to increase the distance of the kicked ball by $34 \%$.
\end{abstract}

\section{INTRODUCTION}

While competitions involve multiple humanoids, individual agents specialization and optimization are an issue for the enhancement of team play. As most RoboCup leagues are based on soccer games to develop robotics, walking and kicking represent two essential skills that playing robots need to master on the field. Walking can be enough to score, because of the possibility of dribbling the ball towards the opponent's goal. Kicking the ball is a very important action in competitive collective soccer games. It can enable higher scoring, but it is also a way to pass the ball between players, which can produce lively and interesting game strategies.

The easiest way to design kicking movements consists of defining fixed keyframes using interpolations techniques (SPL team B-Human [1], SPL team HTWK [2]). Instead of conducting the interpolation inside the joint space, the trajectories can be determined inside the Cartesian space (SPL team Nao Devils [3]), which enables flexibility in the design.

Some methods deal with building omnidirectional kicks while keeping quasi-static balance during the motion, i.e. keeping the projection of the COM within the support polygon. For example Xu et al. [4] used a geometric calculation in the horizontal plane of the farthest-backward reachable point from the hitting point according to the desired kicking direction. Other RoboCup participants like Ferreira et al. from the FC Portugal team [5] built flexible trajectories thanks to path planning based on Bézier curves for the 3D

\footnotetext{
${ }^{a}$ N. Jouandeau is with the Advanced Computer Science Lab (LIASD), Université Paris 8, 2 rue de la Liberté, 93526 Saint-Denis Cedex 02, France, $\mathrm{n}$ at ai.univ-paris8.fr

${ }^{b} \mathrm{~V}$. Hugel is with the Engineering System Lab (LISV), Université de Versailles, 10/12 av. Europe, 78140 Vélizy - France, hugel at lisv.uvsq.fr
}

simulation league. Müller et al. from B-Human [6] also made use of pieces of Bézier curves in the Cartesian space to ensure smooth trajectories in the desired direction.

More recent work developments use the Zero Moment Point (ZMP) [7] to keep the dynamic balance of the robot's executing a kick. The planning of the ZMP trajectory inside the support polygon was employed for biped gaits, while applying a preview controller for increased stability [8], [9]. Yi et al. designed a walk-kick technique using the ZMP preview controller, and applied it on different robotic platforms [10]. Wenk et al. implemented inverse dynamics on the NAO robot to plan the ZMP trajectory, and compared both ZMP planning methods, namely the preview controller and the Linear Quadratic Regulation (LQR) method [11].

In his master thesis, Buckley [12] presented an interesting literature review of human kicking and animated kicking movements. Thanks to parametrised cubic spline trajectories, he proposed to model the swing kick as a pendulum to benefit from the potential energy due to gravity by generating high linear acceleration at the end of the rotated part of the swing movement.

Other developments include the design of a controlledkicking engine that can adapt to a variety of distances angles through a decision method that can select from among a large set of possible kicks [13], and reinforcement learning techniques to deal with penalty kick scenarios [14].

In this work, our aim is not to design accurate nor flexible kicking movements, but to kick and send the ball as far as possible from a fixed position. To achieve this goal, we parametrised the kick with eight parameters, including the rotation angle of the torso and the rotation angle of the foot sole. The rocking of the torso and the rocking of the kicking foot sole must help to transmit more kinetic energy to the ball, and send it even farther away. To find optimized values for the kicking trajectory parameters, we defined an automatized evolution process that is adapted from the Confident Local Optimization techniques (CLOP) [15].

The paper is organized as follows. Section II details the decomposition of the strong kicking movement we developed for the 3D-SSL league using the NAO humanoid model. Section III presents the optimization process designed to make the kick stronger than the manual kick. Section IV details the experimental results. Section V discusses the results before the conclusion.

\section{DESCRIPTION OF THE KICKING MOVEMENT}

A parametrised kick was designed to enable robots to kick the ball far away. Strong kick capabilities offer a serious 

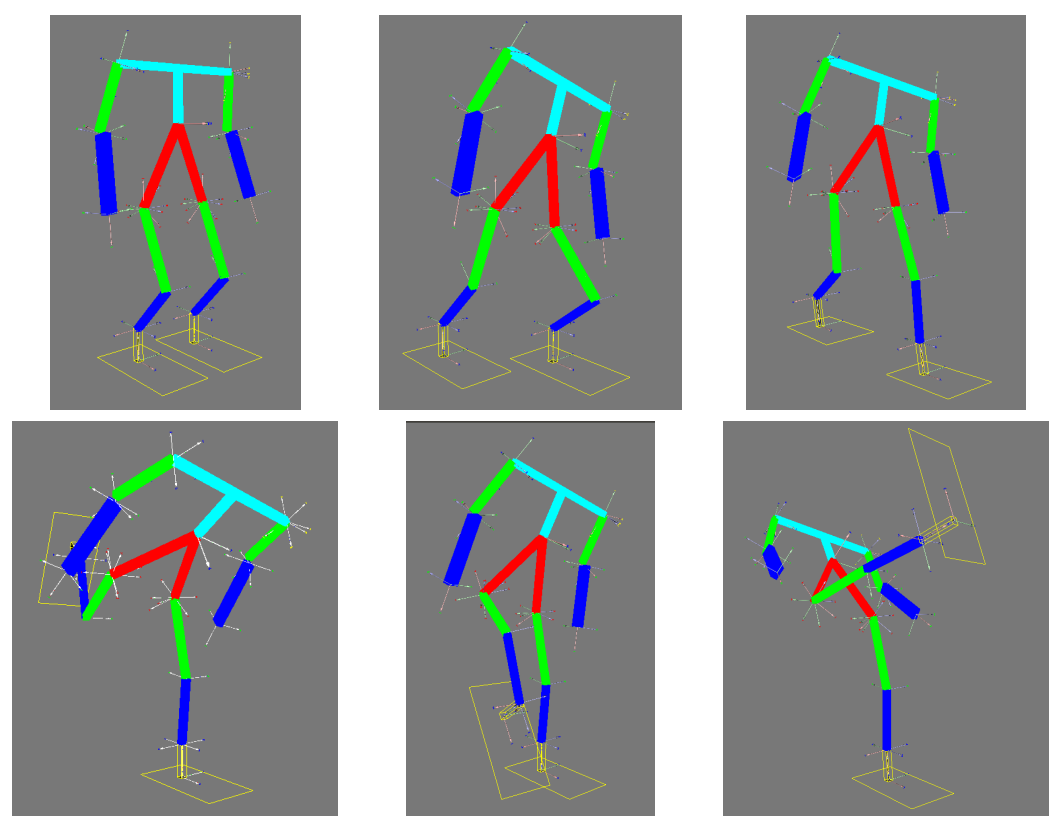

Fig. 1. Decomposition of kicking movement. Initial standing position. End of preparation step. Lift off of leg and body. Backward leg position. Striking leg position. Forward leg position.

advantage to the kick-off team that can send the ball deep into the opponent field.

The parametrised kick consists of the following phases (Fig. 1):

- sway hips to transfer the load above the kicking foot, then lift, swing, and put down the supporting foot. This enables to put the supporting foot next to the ball. Then transfer the load to the supporting foot while tilting the trunk laterally and externally.

- lift the kicking foot while raising the body. The support leg is extended to move the hips higher. This provides the kicking foot with more kinetic energy at the time of striking the ball since the distance between the hip and the foot is increased. The projection of the center of mass remains at the same position.

- put the kicking foot backward while rocking the trunk forward. The final position of the leg is called the backward position.

- put the kicking foot in the position of kick, i.e. the foot toes in contact with the ball, while reaching a fixed inclination of the trunk.

- put the kicking foot forward to accompany the kick movement, while rocking the trunk backward. The final position of the leg is called the forward position.

The rocking of the trunk is possible thanks to the actuation of the hip of the supporting foot. This enables to increase the velocity of the kicking foot at the time of hitting the ball, therefore transmitting a larger amount of kinetic energy to the ball, which permits to send the ball farther away. The kicking movement is parametrised insofar as the positions of the kicking leg in the backward position, in the kicking position and in the forward position can be tuned to optimize the distance covered by the ball after the strike. The parameters
TABLE I

Time Decomposition of Kicking Movement

\begin{tabular}{lr}
\hline Kick phase & Time $[$ sec $]$ \\
\hline sway hips to transfer load to kicking foot & 0.22 \\
lift, swing, put down the other foot & 0.30 \\
tilt the body sideways/transfer load above support foot & 0.60 \\
raise the body and the kicking foot & 0.40 \\
put the kicking foot backward & 0.30 \\
sweep the kicking foot until the striking position & 0.11 \\
sweep the kicking foot forward & 0.11 \\
\hline Full movement & 2.04 \\
\hline
\end{tabular}

for each position are:

1) the longitudinal offset of the foot toes, relative to the position of the support foot,

2) the vertical offset of the foot toes, relative to the position of the support foot,

3) the rotation angle of the torso in the sagittal plane,

4) the rotation angle of the kicking foot sole in the sagittal plane.

Actually the kicking trajectory is defined thanks to a set of three leg configurations defined in the Cartesian space, which makes 12 parameters in total. The inverse kinematics is the same as the one used for the locomotion gaits [16] by the French team L3M-SIM for its participations in the 3D-SSL competitions. The backward-to-strike swing trajectory and the strike-to-forward swing trajectories of foot toe and foot rotation are interpolated linearly, and the time of execution was reduced as much as possible to generate the highest acceleration at the time of hitting the ball.

We assume that the robot's feet are placed adequately close to the ball before triggering the kicking action. We did not implement any COM or ZMP based stabilizer, since it does not matter if the robots falls after striking the ball away. We 
assume that the manual tuning of the trajectory before the strike is enough to prevent the robot from falling too early.

\section{Evolution Process}

The evolution process to find stronger kicks is based on the Confident Local Optimization technique (CLOP) [15]. This technique was already used with success by Jouandeau et al. for the simultaneous evolution of morphological and walking parameters to design morphed players adapted for efficient walk [17] in the framework of the RoboCup 3D Simulation Soccer League.

As usual, the process aims to make a set of bounded parameters evolve through a fitness function. The evolution process repeats the Generate-And-Test algorithm presented in Alg. 1. At each step, the generation of new parameter values is defined with a black box optimizer that takes into account results history (thus trying to maximize future Test results). The evolution process is mainly built on the filling of $\mathcal{L}$ and $\mathcal{H}$ sets over tests. $\mathcal{L}$ defines input parameters with their bounds and $\mathcal{H}$ defines the history set where previous results are collected. At each step of the evolution, the optimization process computes an accurate score over all previous steps that are estimated less confident than the mean of all steps. This iterative maximum-optimization process has been experimentally shown to be less time consuming than classical regression methods for smooth problem optimization; it does not need any reference execution as samples are iteratively selected according to their average win rate. Since the objective consists of finding stable moves, we believe that the smooth optimization of expert parameters is a promising policy. One evolution step corresponds to $k$ trials. At the end of each step, the decision function pickOut states if the result is better, equivalent or worst than the best known result. Initiated with expert knowledge, $\nu^{\prime}$ stores the best known values. The evolution process is implemented as an anytime interruptible algorithm. When the time elapsed is considered as sufficient to produce an interesting solution, the evolution process is instantaneously interrupted and the resulting input parameters are returned.

Applied to kick optimization, the pickOut function (Alg. 2) differentiates the strongest kick according to some constant values. All these evaluation functions make use of the three constant values called $\alpha, \beta$ and $\gamma$ (respect. equal to 31 and 0.7$)-\alpha$ is the nearness factor from the best known result $-\beta$ is the similarity factor from the nearness factor test with a different threshold $-\gamma$ is the stability factor, that is narrowed by the previous best known result. The SUCCESS_RATE allows keeping the process evolution close to stable regions (no fall). It was fixed to 0.75 . This success rate prevents $m$ from taking the value of 0 . The average and standard deviations are computed between successful trials. In the strong kick evolution, the goal is to kick as far as possible. The parametrised type $T$ is not used here but allows to address other types of evolution.

Inside the Test function, the pickOut function compares $\nu$ with $\nu^{\prime}$ and returns three possible values BEST

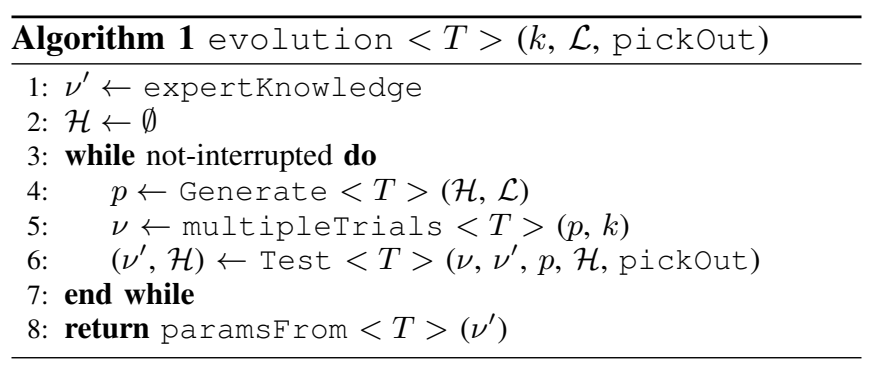

TABLE II

VAlues of Parameters Resulting From Manual Tuning

\begin{tabular}{lccc}
\hline Parameter & Value & Rin & $\max$ \\
\hline \multicolumn{4}{c}{ Backward position } \\
\hline longitudinal toe offset & $-0.140[\mathrm{~m}]$ & -0.240 & -0.040 \\
vertical toe offset & $0.025[\mathrm{~m}]$ & 0.001 & 0.300 \\
angle of torso & $-50.0[\mathrm{deg}]$ & -80.0 & 0.0 \\
angle of foot sole & $73.0[\mathrm{deg}]$ & 0.0 & 110.0 \\
\hline \multicolumn{4}{c}{ Position at strike } \\
\hline longitudinal toe offset & $0.220[\mathrm{~m}]$ & 0.010 \\
vertical toe offset & $0.007[\mathrm{~m}]$ & 0.001 & 0.300 \\
angle of torso & $-15.0[\mathrm{deg}]$ & -45.0 & 0.100 \\
angle of foot sole & $40.0[\mathrm{deg}]$ & 0.0 & 110.0 \\
\hline \multicolumn{4}{c}{ Forward Position $(\mathrm{end} \mathrm{of} \mathrm{sweeping}$ motion) } \\
\hline longitudinal toe offset & $0.300[\mathrm{~m}]$ & 0.100 \\
vertical toe offset & $0.210[\mathrm{~m}]$ & 0.050 & 0.400 \\
angle of torso & $45.0[\mathrm{deg}]$ & 0.0 & 0.300 \\
angle of foot sole & $-65.0[\mathrm{deg}]$ & -110.0 & 110.0 \\
\hline
\end{tabular}

(which implies $\nu^{\prime} \leftarrow \nu$ ), EQUAL and WORST. $\mathcal{H}$ is updated at each evolution iteration.

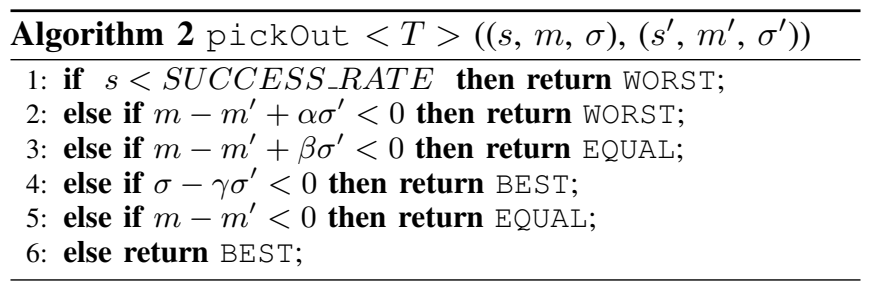

The pickOut function (Alg. 2) uses the 3 subsets $s, m$ and $\sigma$ from $\nu$ (respect. $s^{\prime}, m^{\prime}$ and $\sigma^{\prime}$ from $\nu^{\prime}$ ), that identify the number of success $s$, the average distance $m$ between the final ball and the kicker's positions and its standard deviation $\sigma$. In the test steps of Alg. 2, $s$ and $m$ are considered independently. In order to stay close to real robot motion, we limit the falls thanks to the SUCCESS_RATE threshold. Nevertheless, kicks that produce a 0.25 fall rate or less can be selected if they generate more powerful moves.

\section{EXPERIMENTS}

The humanoid robot used is the NAO robot of the RoboCup 3D Soccer Simulation League (Fig. 5).

Table II gives the parameter values obtained through manual tuning. These parameters allow executing a kick that can send the ball up to $7.5[\mathrm{~m}]$. The duration of the kick is $2.04[\mathrm{sec}]$. The distance between ankles in the initial position is $0.1[\mathrm{~m}]$. Before initiating the kicking motion, the robot bends its torso $17[\mathrm{deg}]$ laterally on the side of the support foot. 


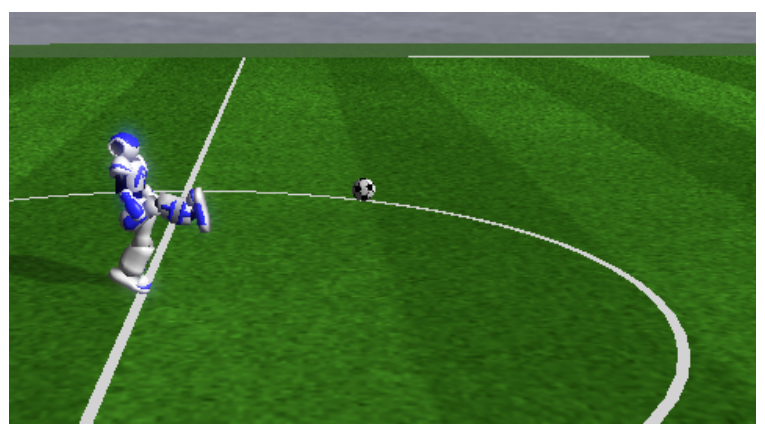

Fig. 2. A kicking motion with simspark.

We tried 3 evolutions named $\mathrm{C} 1, \mathrm{C} 2$, and $\mathrm{C} 3$ :

- C1 : is composed of all 6 angular parameters, i.e. angles of torso and foot sole for the backward, strike and forward positions,

- C2 : is composed of the $\mathrm{X}-$ and-z toe offsets at strike only,

- $\mathrm{C} 3$ : is composed of all the 8 kicking parameters defined for $\mathrm{C} 1$ and $\mathrm{C} 2$.

$\mathrm{C} 1$ and $\mathrm{C} 3$ are applied over the standard parameters. $\mathrm{C} 2$ is complementary to $\mathrm{C} 1$. After $\mathrm{C} 1$, we optimized $\mathrm{C} 2$ to produce a more precise kick in a reduced parametrised space. We did not take into account the $\mathrm{x}-\mathrm{z}$ parameters of the backward and forward positions because they are assumed to have less influence on the result, and because a reduced set of parameters is better to speed up the optimization process.

\section{Results}

Tests are carried out on a single computer that runs the simulation server rcssserver $3 d$ [18], our parametrised player agent rcssagent $3 d-13 m$ [19] and a coach that is responsible for starting each trial. To compare the results, all evolution processes were stopped after 48 hours, which makes approximately one thousand iterations.

Table III gives the parameter values obtained for each evolution. Figure 3 shows the results for 500 iterations of $\mathrm{C} 2$. The best results are represented in green, equivalent results in blue and worst results in red. Figure 3 shows the distribution of values in the sub-space (xToe, zToe) and the nature of the results. Concentration values close to $\mathrm{xToe}=0.2$ at the bottom show the mutual dependence between these two parameters in characterizing nice kicking moves. Figure 4 shows the distribution during the first 100 iterations, and Fig. 5 during the last 200 iterations. It shows the evolution turning from unstable (at the beginning) to stable (at the end) while kicking results appear to be better than previous ones over the successive iterations.

$\mathrm{xT}$ oe varies between -0.01 and $-0.3[\mathrm{~m}]$ on the horizontal x-axis. zToe varies between 0.001 and $0.1[\mathrm{~m}]$ on the vertical y-axis. It shows that the optimization process leads to a confined area around $[0.2 ; 0.01]$.

After the evolution process, resulting parameters are tested carefully. Figure 6 shows the final ball coordinates over 50 runs in the global coordinate system for each class $\mathrm{C} 1, \mathrm{C} 2$,
TABLE III

PARAMETERS SETS CLASSIFICATION AND EVOLUTIONS RESULTS

\begin{tabular}{|c|c|c|c|c|c|c|}
\hline \multicolumn{2}{|c|}{ Classes } & \multirow{2}{*}{\multicolumn{3}{|c|}{$\begin{array}{l}\text { Parameter description } \\
\text { angle of torso } \\
\text { angle of foot sole }\end{array}$}} & \multirow{2}{*}{$\begin{array}{l}\text { Parameter } \\
\text { angT0 } \\
\text { angFS0 }\end{array}$} & \multirow{2}{*}{$\begin{array}{l}\text { Position } \\
\text { Backward }\end{array}$} \\
\hline \multirow{4}{*}{ C3 } & \multirow{3}{*}{$\mathrm{C} 1$} & & & & & \\
\hline & & $\begin{array}{l}\text { ang } \\
\text { ang }\end{array}$ & $\begin{array}{l}\text { of torso } \\
\text { ef foot sole }\end{array}$ & & $\begin{array}{l}\text { angT2 } \\
\text { angFS2 }\end{array}$ & Forward \\
\hline & & $\begin{array}{l}\text { ang } \\
\text { ang }\end{array}$ & $\begin{array}{l}\text { e of torso } \\
\text { e of foot sole }\end{array}$ & & $\begin{array}{l}\text { angT1 } \\
\text { angFS1 }\end{array}$ & \multirow{2}{*}{$\begin{array}{l}\text { At strike } \\
\text { (middle of } \\
\text { sweeping } \\
\text { motion) }\end{array}$} \\
\hline & $\mathrm{C} 2$ & $\begin{array}{l}\text { lon } \\
\text { ver }\end{array}$ & $\begin{array}{l}\text { itudinal toe off } \\
\text { cal toe offset }\end{array}$ & & $\begin{array}{l}\text { xToe } \\
\text { zToe }\end{array}$ & \\
\hline & \multicolumn{2}{|c|}{ Parameter } & C1 & & $\mathrm{C} 2$ & $\overline{53}$ \\
\hline & \multicolumn{2}{|c|}{ angT0 } & $-43.5[$ deg $]$ & & \multicolumn{2}{|c|}{$-58.6[\mathrm{deg}]$} \\
\hline & \multicolumn{2}{|c|}{ angFS0 } & 98.1[deg] & & \multicolumn{2}{|c|}{$96.5[\mathrm{deg}]$} \\
\hline & \multicolumn{2}{|c|}{ angT1 } & $-28.5[\mathrm{deg}]$ & & \multicolumn{2}{|c|}{$-14.2[\mathrm{deg}]$} \\
\hline & \multicolumn{2}{|c|}{ angFS1 } & $31.2[\mathrm{deg}]$ & & \multicolumn{2}{|c|}{$92.1[\mathrm{deg}]$} \\
\hline & \multicolumn{2}{|c|}{ xToe } & & \multicolumn{2}{|c|}{$0.212[\mathrm{~m}]$} & $0.266[\mathrm{~m}]$ \\
\hline & \multicolumn{2}{|c|}{ zToe } & & & $04[m]$ & $0.084[\mathrm{~m}]$ \\
\hline & \multicolumn{2}{|c|}{ angT2 } & $62.5[\operatorname{deg}]$ & & \multicolumn{2}{|c|}{$70.4[\mathrm{deg}]$} \\
\hline & \multicolumn{2}{|c|}{ angFS2 } & $-56.6[\mathrm{deg}]$ & & \multicolumn{2}{|c|}{$104.1[\mathrm{deg}]$} \\
\hline
\end{tabular}

and $\mathrm{C} 3$, and for expert parameters. For each experiment, the initial position of the robot on the field is considered as the origin of the global coordinate system.

Corresponding averages and standard deviations are detailed in table IV. It shows that $\mathrm{C} 1$ resulting parameters produce a stronger kick that sends the ball 1.34 times farther than expert parameters. Similar results are obtained for the C3 parameters. By using C2 parameters, the lateral deviation of the ball can be reduced as the related standard deviation is 2 times less than $\mathrm{C} 1$, and 4.7 times less than $\mathrm{C} 3$ results. It shows that dividing the $\mathrm{C} 3$ evolution into $\mathrm{C} 1$ followed by $\mathrm{C} 2$ produces a more accurate motion, considering a comparable optimization process with an equivalent number of iterations.

\section{DiscusSION}

This study shows that the proposed evolution process based on the CLOP technique is useful to increase the kicking range of the ball. The kick swing was decomposed into three positions. Each position was defined by two angles, torso and foot sole, and two coordinates of the foot toe, namely $\mathrm{x}$ and $\mathrm{z}$. The evolution process took into account eight parameters, including the angle parameters of the three positions, and the $\mathrm{x}, \mathrm{z}$ parameters of the strike position. The positions of the toe in the backward and the forward positions were not used as evolving parameters in the optimization process. These position parameters could be added in the process to change the curvature of the swing trajectory, which may have an influence on the kick strongness.

In addition, cubic splines trajectories instead of linear interpolation for the swing motion could be useful to get a highest velocity at the moment of hitting the ball.

The process assumed that the robot accurately placed its feet in a fixed position with respect to the ball. In particular the lateral position of the foot toe remains fixed. The variation of the lateral coordinate of the foot toe was not taken into account in the evolution process, which explains the deviation of the ball from the longitudinal axis of the robot. However the lateral position of the toe can be adjusted 
to modify the kicking direction so as to hit the ball in the center, which can provide more flexibility to the kicking motion.

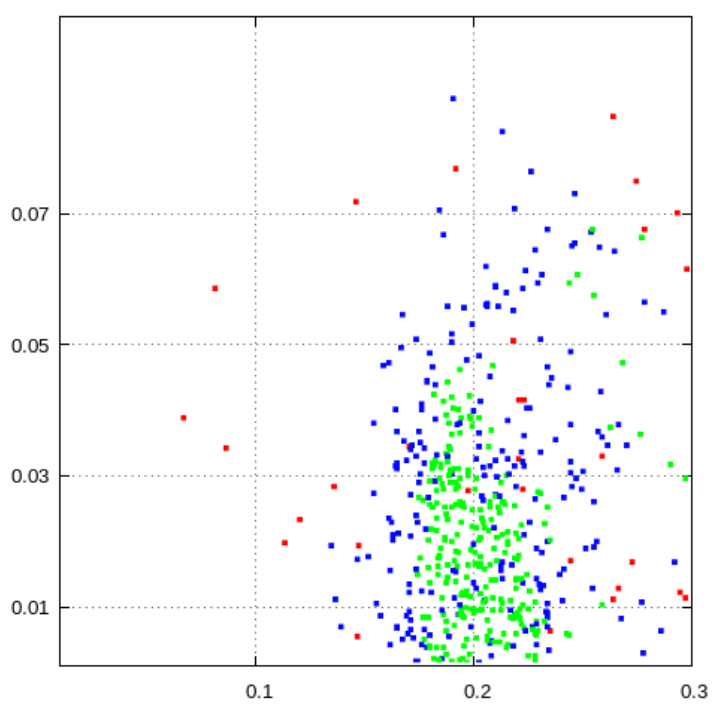

Fig. 3. Evolution of xToe and zToe values [m] of Position at strike over 500 iterations of $\mathrm{C} 2$.

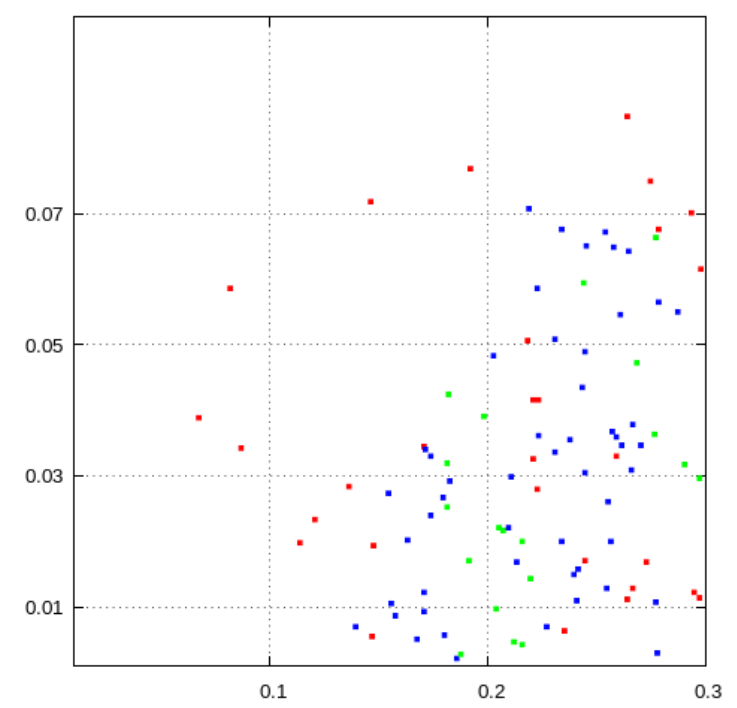

Fig. 4. Evolution of xToe and zToe values [m] of Position at strike during the first 100 iterations of $\mathrm{C} 2$.

The swing motion includes a rocking motion of the torso and a rotation of the foot sole with the aim to increase the velocity of the foot when striking the ball. The rocking motions come from the activation of the hip pitch joint and the ankle pitch joint. The hip of the NAO robot also features a yaw joint at $45[\mathrm{deg}]$. The lateral component of this joint could be used in conjunction with the pitch joint to increase the velocity of the swing motion at the time of strike. Then the swing motion will not be limited to the sagittal plane but will follow a $3 \mathrm{D}$ curved trajectory. This is

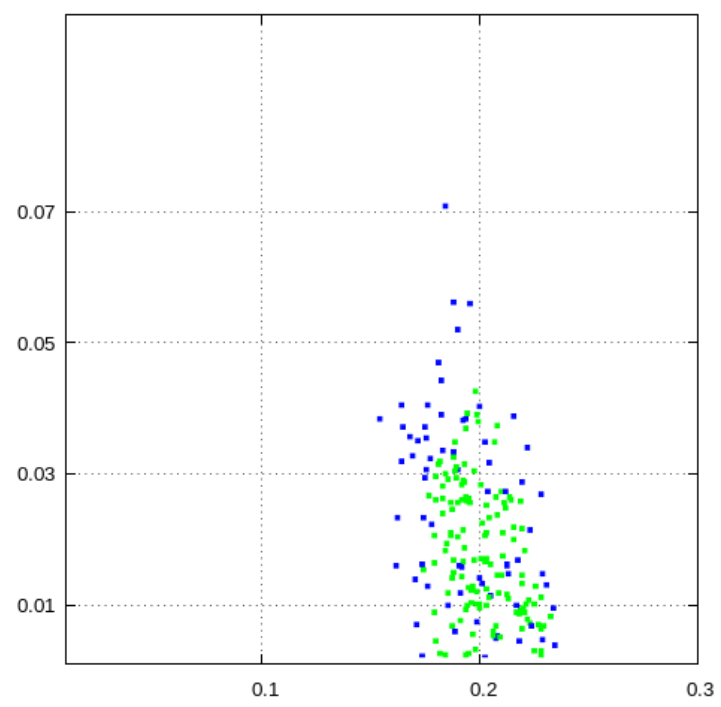

Fig. 5. Evolution of xToe and zToe values [m] of Position at strike during the last 200 iterations of $\mathrm{C} 2$.

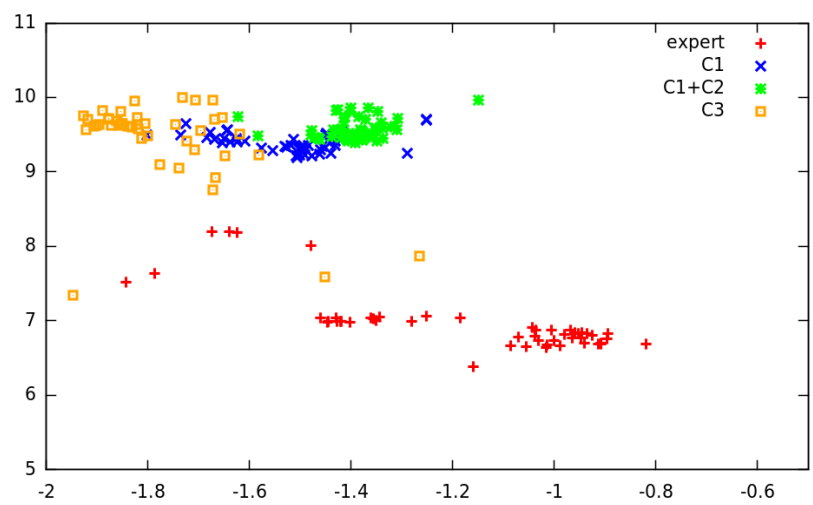

Fig. 6. Horizontal ball position coordinates $[\mathrm{m}]$ after a kick with expert, $\mathrm{C} 1, \mathrm{C} 2$ and $\mathrm{C} 3$ evolutions.

a future improvement that requires a specific calculation to synchronize both the movements of the kicking leg and the torso because the yaw joints of the NAO are coupled.

\section{CONCLUSION}

This paper presented an optimization process designed to increase the kicking range of humanoid players in the 3D-soccer simulation league. The proposed anytime process allowed improving the values tuned manually to win $34 \%$ ball kicking range. The optimization process was divided into a two-classes sequential process, that produces more accurate motion. More flexible motion remains to be develop, to produce adaptable kicks capable of sending the ball at various distances. This could be achieved by varying parameters such as the foot sole angle. 
TABLE IV

AVERAGE AND STANDARD DEVIATIONS

\begin{tabular}{|l|ll|ll|ll|}
\hline Classes & \multicolumn{2}{|l|}{ longitudinal } & \multicolumn{2}{l|}{ lateral [m] } & \multicolumn{2}{l|}{ distance [m] } \\
\hline Expert & 6.974 & 0.401 & -1.181 & 0.265 & 7.076 & 0.432 \\
\hline C1 & 9.389 & 0.127 & -1.525 & 0.114 & 9.512 & 0.130 \\
\hline C2 & 9.585 & 0.143 & -1.392 & $\mathbf{0 . 0 7 0}$ & $\mathbf{9 . 6 8 6}$ & 0.140 \\
\hline C3 & 9.383 & 0.617 & -1.861 & 0.329 & 9.572 & 0.609 \\
\hline
\end{tabular}

\section{REFERENCES}

[1] T. Röfer, T. Laue, J. Müller, J. O. Bösche, A. Burchardt, E. Damrose, K. Gillmann, C. Graf, T.J. de Haas, A. Härtl, A. Rieskamp, A. Schreck, I. Sieverdingbeck and J.H. Worch. BHuman Team Report and Code Release, 2009. Available online: http://www.b-human.de/file_download/26/bhuman09_c oderelease.pdf.

[2] R. Tilgner, T. Reinhardt, D. Borkmann, T. Kalbitz, S. Seering, R. Fritzsche, C. Vitz, S. Unger, S. Eckermann, H. Müller, M. Bellersen, M. Engel and M. Wünsch. Team research report 2011 Nao-Team HTWK Leipzig, 2011. Available online: http://robocup.imn.htwk-leipzig.de/documents/rep ort2011.pdf.

[3] S. Czarnetzki, S. Kerner and D. Klagges. Combining key frame based motion design with controlled movement execution. Robot Soccer World Cup XIII, Lecture Notes in Computer Science, 2010, vol. 5949, pp. 5868.

[4] Y. Xu and H. Mellmann. Adaptive Motion Control: Dynamic Kick for a Humanoid Robot. KI 2010: Advances in Artificial Intelligence, Lecture Notes in Computer Science,2010, vol. 6359, pp. 392-399.

[5] R. Ferreira, L.P. Reis, A.P. Moreira and N. Lau. Development of an Omnidirectional Kick for a NAO Humanoid Robot. Lecture Notes in Computer Science, Advances in Artificial Intelligence - IBERAMIA 2012, vol. 7637, pp. 571-580.

[6] J. Müller, T. Laue and T. Röfer. Kicking a ball - modeling complex dynamic motions for humanoid robots. Robot Soccer World Cup XIV. Lecture Notes in Artificial Intelligence, vol. 6556, 2011, pp. 109-120.

[7] M. Vukobratović and B. Borovac. Zero-moment point - thirty five years of its life. International Journal of Humanoid Robotics, 01(01), 2004, pp. 157-173.

[8] J.J. Alcaraz-Jiménez, D. Herrero-Pérez and H. Martíez-Barberá. Motion Planning for Omnidirectional Dynamic Gait in Humanoid Soccer Robots. J. of Physical Agents, Vol. 5(1), 2011, pp. 25-34.

[9] S. Kajita, F. Kanehiro, K. Kaneko, K. Fujiwara and K.H.K. Yokoi. Biped walking pattern generation by using preview control of zero- moment point. Proc. of the IEEE Int. Conf. on Robotics and Automation, 2003, pp. $1620-1626$.

[10] S.J. Yi, S. McGill, and D.D. Lee. Improved Online Kick Generation Method for Humanoid Soccer Robots. 8th workshop of humanoid soccer robots, Humanoids Conference, 2013.

[11] F. Wenk and T. Röefer. Online Generated Kick Motions for the NAO Balanced Using Inverse Dynamics. Robot Soccer World Cup XVII, Lecture Notes in Artificial Intelligence, 2013.

[12] A. Buckley. Humanoid Robot Soccer Locomotion, and Kick Dynamics, Open Loop Walking, Kicking and Morphing into Special Motions on the Nao Robot. Master Thesis. National University of Ireland Maynooth, 2013.

[13] S. Barrett, K. Genter, T. Hester, M. Quinlan and P. Stone. Controlled Kicking under Uncertainty. The Fifth Workshop on Humanoid Soccer Robots (HSR-10), 2010.

[14] T. Hester, M. Quinlan and P. Stone. Generalized model learning for Reinforcement Learning on a humanoid robot. IEEE Int. Conf. on Robotics and Automation, 2010, pp. 2369-2374.

[15] R. Coulom. CLOP: Confident Local Optimization for Noisy Black-Box Parameter Tuning, In Advanced Computer Games (ACG), volume 7168 of Lecture Notes in Computer Science, page 146-157. Springer, 2011.

[16] V. Hugel and N. Jouandeau. Automatic generation of humanoid's geometric model parameters. In Proceedings of 17th annual RoboCup International Symposium 2013, (RCUP 2013).

[17] N. Jouandeau and V. Hugel. Simultaneous evolution of leg morphology and walking skills to build the best humanoid walker. In IEEE-RAS International Conference on Humanoid Robots 2013, 8th Workshop on Humanoid Soccer Robots, (WHSR 2013).
[18] O. Obst and M. Rollmann. Spark - A Generic Simulator for Physical Multi-Agent Simulations. In Multiagent System Technologies, Lecture Notes in Computer Science, vol. 3187, pp. 243-257, 2004.

[19] N. Jouandeau, V. Hugel and T. Da Costa, 3DSSL Team Description Paper. In Robocup 3D Soccer Simulation League 2013, (RCUP-2013). 